Orthopäde 2022 · 51:246-250

https://doi.org/10.1007/s00132-021-04205-6

Angenommen: 24. November 2021

Online publiziert: 6 . Januar 2022

(c) Der/die Autor(en) 2022

\section{Insuffizienzfraktur der Klavikula nach Implantation einer inversen Schulterendoprothese}

Laura Elisa Streck · Lothar Seefried · Franca Genest · Thomas Reichel ·

Maximilian Rudert · Kilian Rueckl

Lehrstuhl für Orthopädie der Julius-Maximilians-Universität Würzburg, Orthpädische Klinik König-LudwigHaus, Würzburg, Deutschland

\title{
Zusammenfassung
}

Wir stellen den seltenen Fall einer Insuffizienzfraktur der Klavikula nach Implantation einer inversen Schulterendoprothese (RSA) vor. Als Ursache solcher Frakturen wird eine vermehrte Zugbelastung durch den Musculus deltoideus nach RSA diskutiert. In den wenigen verfügbaren Fallberichten zeigten die betroffenen Patienten deutliche Funktionseinschränkungen. Die Versorgung erfolgte im vorliegenden Fall mit Plattenosteosynthese. Trotz intraoperativ gutem Korrekturergebnis kam es im Verlauf ohne Trauma zum Osteosyntheseversagen mit weiterer Dislokation der Fraktur.

\section{Schlüsselwörter}

Glenohumeralgelenk - Osteoporose - Osteosynthese, Fraktur · Postoperative Komplikationen . Schulterendoprothetik

\section{Einleitung}

Die Implantation einer inversen Schulterendoprothese (RSA) ist bei Patienten mit fortgeschrittener Defektarthropathie, Pseudoparalyse und ausgeprägten Glenoiddefekten eine erfolgreiche Therapieoption [3, 13, 14]. Mit zunehmender Anzahl der Primärimplantationen steigt jedoch auch die Zahl der Komplikationen, welche in 6-50\% der Fälle beschrieben werden [2, 14]. Bekannte Probleme sind aseptische Lockerungen, skapuläres Notching, periprothetische Infektionen oder Insuffizienzfrakturen des Akromions $[2,3,13,14]$. Eine sehr seltene aber nicht weniger relevante Komplikation stellt die Insuffizienzfraktur der Klavikula nach Implantation einer RSA da [1, 8, 9]. Der aktuelle Fallbericht zeigt die Schwierigkeiten der Behandlung dieser Komplikation und diskutiert die verfügbare Literatur.

\section{Anamnese}

Eine 69-jährige Patientin stellte sich mit progredienten Schmerzen und Bewegungseinschränkung der linken Schulter ohne Ansprechen auf konservative Therapie in unserer Klinik vor. Bei vorbekannter Defektarthropathie (Hamada 4b) offenbarte die konventionelle Röntgenaufnahme eine Schulterluxation (• Abb. 1).

Die Patientin hatte multiple Vorerkrankungen. Sie litt an rheumatoider Arthritis und einem fortgeschrittenen Morbus Parkinson (Erstdiagnose 1990) mit rezidivierenden Stürzen. Hinzu kam eine multifaktorielle Osteoporose nach langjähriger Vorbehandlung mit Glukokortikoiden und MTX mit mehreren vorangegangenen, pathologischen Frakturen seit 2005. Ab 2007 erfolgte dahingehend eine medikamentöse Therapie mit wechselnden Präparaten, seit 2016 mit Denosumab. Darüber hinaus bestanden ein knochenmarkzytologisch gesichertes myelodysplastisches Syndrom mit multilinearer Dysplasie mit zytogenetischem (FISH) Deletionsnachweis 7p15 und molekulargenetisch nachgewiesenen BCOR- und DNMT3A-Mutationen sowie klinisch führender Thrombozytopenie. Zudem bestand ein erhöhtes kardiovaskuläres Risiko bei arterieller Hypertonie und Zustand nach tiefer Beinvenenthrombose sowie Lungenembolie. Nach differenzial- 

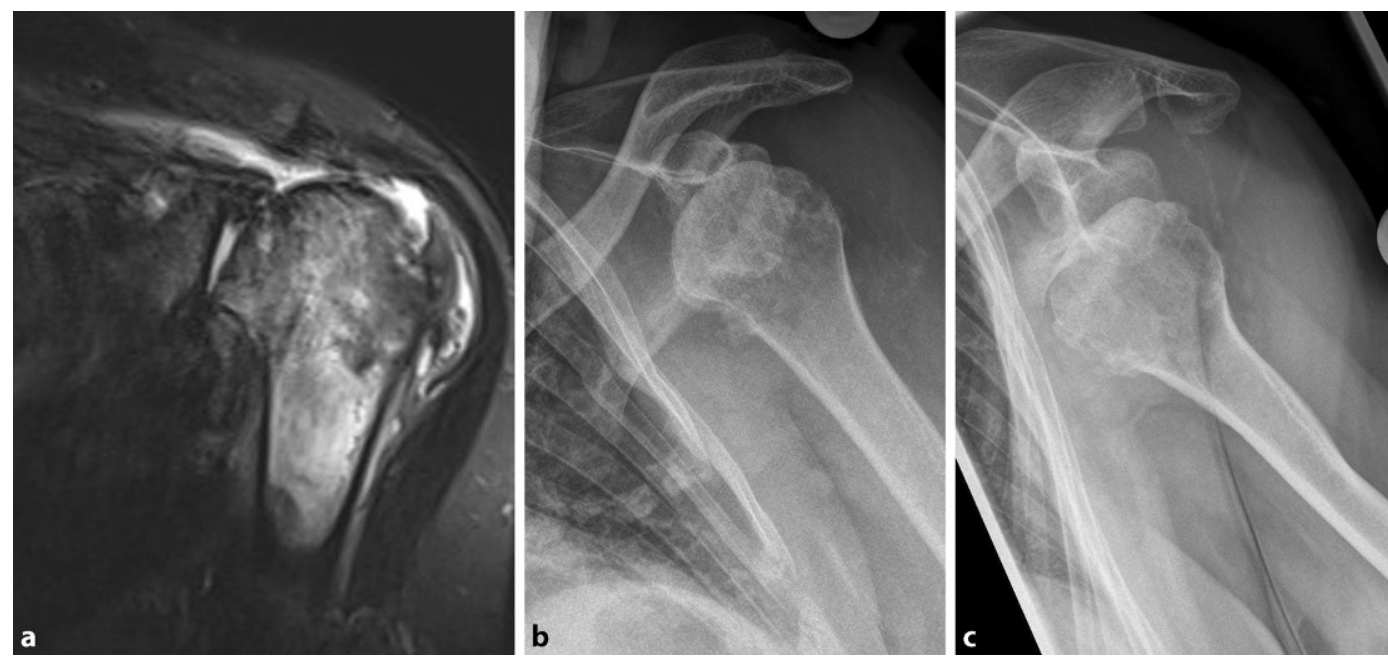

Abb. $1<$ a Koronarer Schnitt einer MRT der linken Schulter. Zu erkennen ist die Komplettruptur der Supraspinatussehne mit weiter Retraktion des Sehnenstumpfes (Patte 3). b, c Röntgenaufnahmen der linken Schulter in zwei Ebenen. Es bestand eine Schulterluxation bei Defektarthropathie

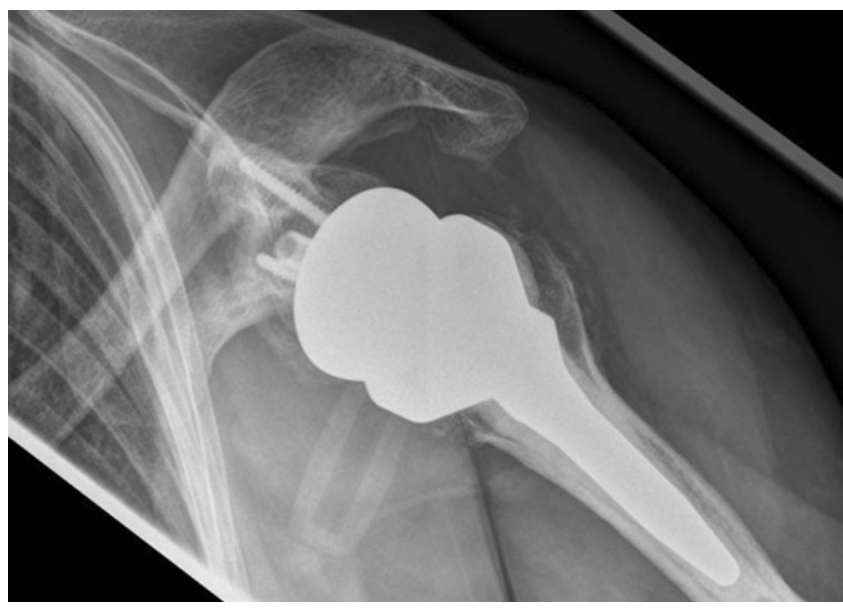

Abb. 2 ム Postoperative Röntgenaufnahme im axialen Strahlengang nach Implantation einer inversen Schulterendoprothese und knöchernem Aufbau des Glenoids

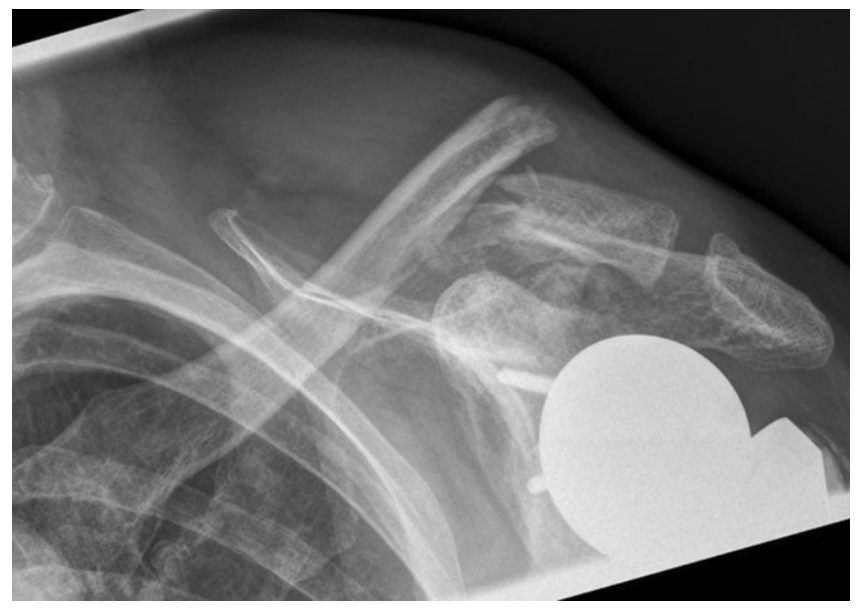

Abb. 3 ॥ Die Röntgenaufnahme im anteroposterioren Strahlengang zeigte eine laterale Klavikulafraktur (Robinson 3B1). Die mit abgebildeten Anteile der Endoprothese lagen unverändert ein therapeutischer Abwägung wurde daher auf eine osteoanabole Intervention mit Teriparatid/Romosozumab verzichtet.

Aufgrund der ausgeprägten Schmerzen und der massiven Einschränkung der Alltagsaktivitäten wurde die Indikation zur Implantation einer RSA gestellt. Es erfolgte die komplikationslose Implantation einer Tornier Aequalis Reversed II Prothese (Wright Medical Group N. V., Memphis, TN, USA) mit zementiertem Schaft. Bei anteriorer Defektsituation des Glenoids (Walch Typ D) erfolgte die Rekonstruktion durch Fixierung des hälftigen Humeruskopfes mit Magnesiumschrauben (Magnezix ${ }^{\circledR}$, Syntellix AG, Hannover, Deutschland), gefolgt von der Implantation einer Basisplatte mit langem Post. Die postoperative Röntgenbildgebung ist in - Abb. 2 dargestellt. Postoperativ erfolgte die Ruhigstellung in der Schulter-ArmOrthese sowie die passive Mobilisierung für 6 Wochen. Ab der 7. Woche erfolgte zunehmend auch die aktive Mobilisierung, ab der 9. Woche wurde Physiotherapie mit leichten Widerständen begonnen. Zunächst zeigte sich ein regelrechter postoperativer Verlauf, die Patientin war schmerzfrei. 18 Wochen postoperativ traten nach physiotherapeutischer Beübung der Abduktion zunehmende Schmerzen im Bereich der Klavikula auf.

\section{Befund}

Klinisch zeigte sich eine Stufenbildung der lateralen Klavikula mit irregulärer Beweglichkeit und Krepitationen. Es bestanden keine Einschränkungen der Neurologie oder Durchblutung.

\section{Diagnose}

Röntgenologisch bestätigte sich die Verdachtsdiagnose einer lateralen Klavikulafraktur (Robinson 3B1, Jäger Breitner III) mit kranialer Dislokation des medialen Fragments (- Abb. 3).

\section{Therapie und Verlauf}

Es erfolgte die komplikationslose Versorgung mittels offener Reposition und winkelstabiler Plattenosteosynthese (5-Loch LCP mit EXT; Synthes Inc., West Chester, PA, USA). Die postoperative Röntgenkontrolle ist in - Abb. 4 dargestellt. Die osteologische Medikation wurde unter Berücksichtigung aktueller Laborparameter und Knochendichtemessungen nochmals durch einen Osteologen (DVO) reevalu- 

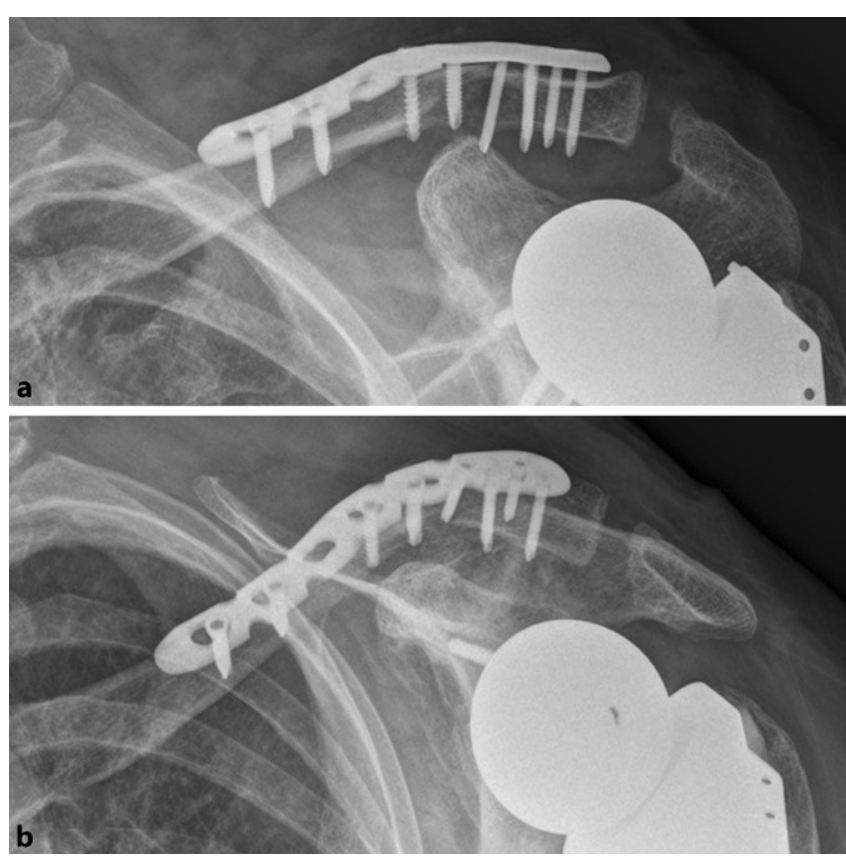

Abb. $4 \Delta$ Röntgenaufnahmen in axialer (a) und anteroposteriorer (b) Projektion nach Versorgung der lateralen Klavikulafraktur mit Plattenosteosynthese. Die vierte und fünfte Schraube von lateral gesehen lagen in der Frakturzone, fassten jedoch beide Fragmente

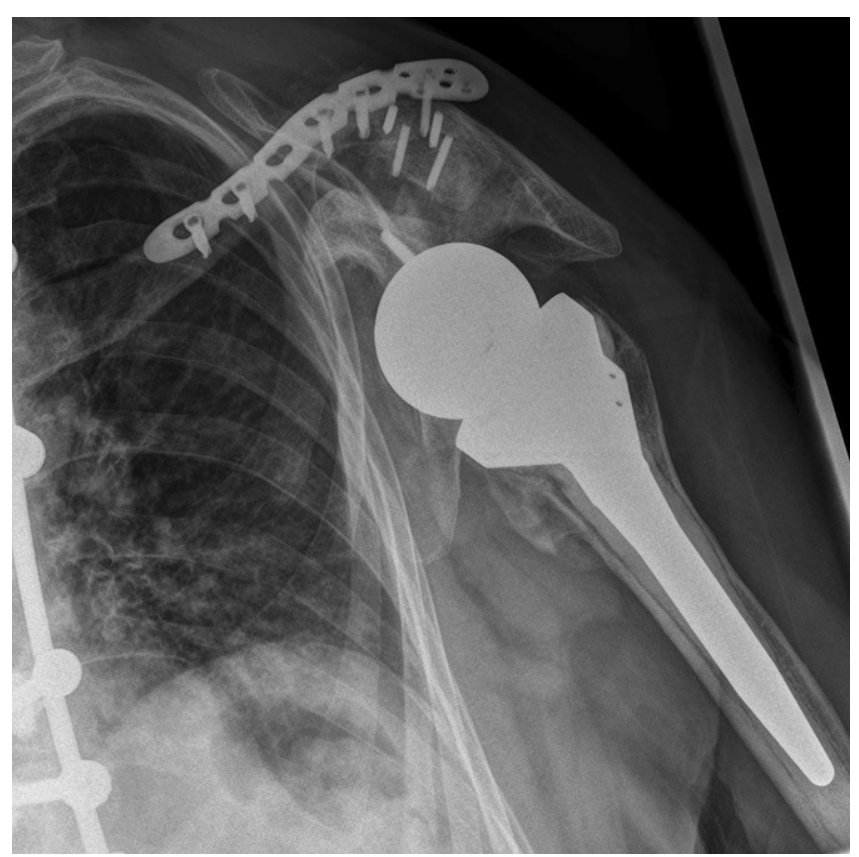

Abb. $5 \Delta$ Röntgenaufnahme der linken Schulter mit Oberarm in modifizierter anteroposteriorer Projektion. Es zeigte sich ein Osteosyntheseversagen mit Dislokation der Platte und Bruch von 4 lateralen Schrauben sowie Dislokation der Fraktur. Die inverse Endoprothese lag weiterhin regelrecht ein iert, hierbei wurde keine Indikation zur Änderung des bestehenden Therapieregimes (Vitamin D, Denosumab) gesehen.

Für die ersten 6 Wochen postoperativ erfolgte die Ruhigstellung in der SchulterArm-Orthese mit passiver Mobilisierung und aktiv assistierter Flexion und Abduktion bis $80^{\circ}$. Bei zunächst regelrechtem Verlauf kam es 5 Wochen postoperativ zu vermehrten Schmerzen. Ein auslösendes Trauma wurde von der Patientin glaubhaft verneint. Röntgenologisch zeigte sich die erneute Dislokation des medialen Fragments nach kranial, 4 laterale Schrauben waren abgebrochen, eine laterale Schraube war aus dem Fragment disloziert. Im medialen Fragment bestand röntgenologisch kein Hinweis auf eine Lockerung, die Prothese lag weiterhin regelrecht ein (• Abb. 5).

Bei multiplen Vorerkrankungen, limitierter Knochenqualität und Thrombozytopenie (14.000 Thrombozyten $/ \mathrm{mm}^{3}$ ) entschieden wir uns gemeinsam mit der Patientin gegen eine Reosyteosynthese mit Hakenplatte und korakoklavikulärer Stabilisierung. In den nachfolgenden Verlaufskontrollen war die Haut- und Weichteilsituation stets unauffällig. Im zeitlichen Verlauf bis 3 Monate post- operativ bestand kein Hinweis auf eine wesentliche sekundäre Dislokation des Osteosynthesematerials oder der Fragmente bei zunehmender Kallusbildung (• Abb. 6). Während präoperativ dauerhaft massive Schmerzen (8/10 Punkte nach Numerischer Rating-Skala) bestanden hatten, wurden die Schmerzen nun mit 1/10 nach Numerischer Rating-Skala angegeben. Die Beweglichkeit nach Auftreten der Komplikation war mit Anteversion bis $30^{\circ}$ und Abduktion $20^{\circ}$ stark eingeschränkt und unverändert zu den präoperativen Befunden. Die Patientin konnte sich jedoch nahezu schmerzfrei auf dem Gehwagen abstützen, sodass die Mobilität der Patientin im Vergleich zur präoperativen Situation deutlich verbessert werden konnte.

Die Patientin wünschte weiterhin keine operative Revision.

\section{Diskussion}

Die RSA wurde seit den ersten Modellen in den 1970er-Jahren konstant weiterentwickelt. Zahlreiche Möglichkeiten zur individuellen Anpassung von Offset, Schaftinklination oder Position der Glenosphäre stehen zur Verfügung. Längst stellt nicht mehr nur die Defektarthropathie eine Indikation zur Implantation einer RSA dar, sondern auch Frakturen mit Ausriss der Tuberkula, Instabilitätsarthrose oder glenoidale Defekte [14]. Insgesamt zeigen sich gute Ergebnisse mit Verbesserung der Schmerzsituation, Beweglichkeit und Funktionalität. Die revisionsfreie Standzeit wird mit $89 \%$ nach 10 Jahren angegeben [5]. Trotz der steten technischen Weiterentwicklung und der insgesamt guten Ergebnisse treten jedoch in bis zu 50\% der Fälle Komplikationen auf. Hierzu zählen insbesondere skapuläres Notching, Lockerungen der Glenoidkomponente, Instabilität der Prothese und periprothetische Infektionen [2].

Akromionfrakturen werden bei bis zu $4 \%$ der Patienten beschrieben [11]. Durch die Verlängerung des Hebelarmes des Musculus deltoideus entsteht eine erhöhte Belastung auf dessen akromialen Ansatz. Insbesondere bei Patienten mit Osteoporose oder rheumatologischer Vorerkrankung kann es zu Insuffizienzfrakturen kommen [10]. Sowohl bei konservativer wie auch operativer Versorgung zeigen sich hohe Pseudarthroseraten. Die langfristigen funktionellen Ergebnisse von Patienten mit Akromionfraktur sind 

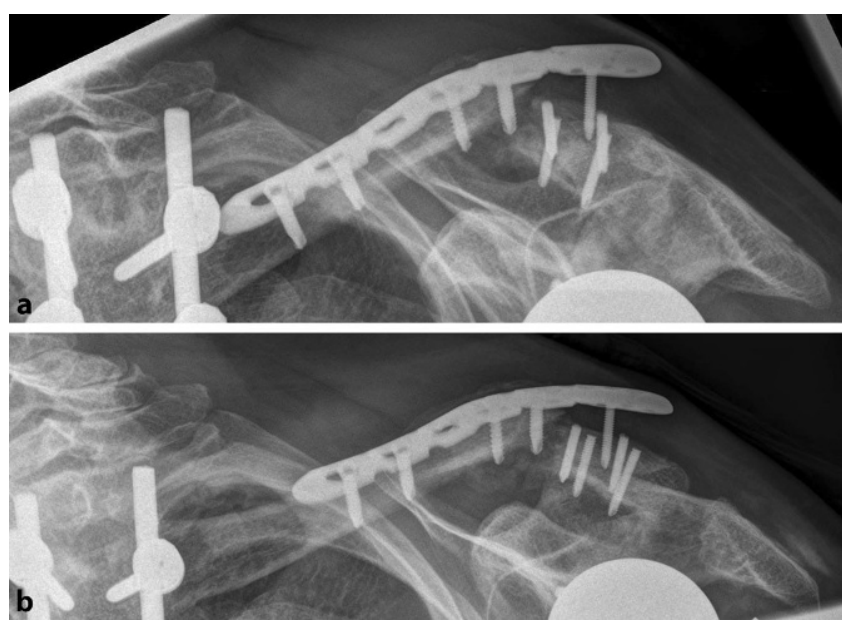

Abb. $6<$ Röntgenaufnahmen in anteroposteriorer Projektion. Im zeitlichen Verlauf 6 Wochen (a) und 3 Monate (b) postoperativ blieben die Positionen von Fraktur und Osteosynthesematerial im Wesentlichen stabil, die Kallusbildung nahm $\mathrm{zu}$

tendenziell schlechter als bei anderen Patienten [11].

Insuffizienzfrakturen der Klavikula stellen dagegen eine Rarität dar. Soweit den Autoren bekannt ist, wurden bislang lediglich 3 Fälle beschrieben $[1,8,9]$. Betroffen waren Patientinnen zwischen 64 und 90 Jahren. Die Frakturen lagen im lateralen Drittel (1 Fall) beziehungsweise am Übergang vom mittleren zum lateralen Drittel der Klavikula (2 Fälle) und traten 6 Wochen bis 10 Monate postoperativ auf. Bei 2 Patientinnen ereignete sich die Fraktur zeitnah nach Aufnahme der aktiven Mobilisierung [1, 8]. Die Versorgung erfolgte in allen Fällen zunächst konservativ mit Ruhigstellung. Bei 2 Patientinnen kam es zur sekundären Dislokation [1, 9]. In beiden Fällen wurde die Indikation zur Plattenosteosynthese gestellt, eine Patientin lehnte die Operation ab [9]. In allen Fällen zeigte sich auch über ein Jahr nach Fraktur noch eine deutliche Funktionseinschränkung [1, 8, 9].

Ätiologisch scheint eine Ermüdungsfraktur durch die erhöhte Zugbelastung des Musculus deltoideus, Pars clavicularis, vergleichbar den Frakturen der Spina scapulae und des Akromions, plausibel. Auch eine vermehrte Belastung der Klavikula bei Kraftübertragung über das Akromioklavikulargelenk ist denkbar, diese besteht insbesondere bei Abduktionsbewegungen. Als weiterer Mechanismus wird zudem eine bereits präoperativ bestehende Vorschädigung der Klavikula diskutiert $[1,8,9]$. Kim et al. sahen die Möglichkeit, dass bei Läsion des Musculus subscapularis durch eine anteriore Migration des Humeruskopfes auch vermehrter Stress auf die
Klavikula wirken kann [8]. Eine ausgiebige Literaturrecherche konnte keine Studien finden, welche entsprechende biomechanische Überlegungen für Patienten mitRSA überprüft haben. Im aktuellen Fall bestand präoperativ kein Hinweis auf eine Pathologie der Klavikula.

Die Begleiterkrankungen, konkret der Morbus Parkinson mit erhöhtem Sturzrisiko und die rheumatoide Arthritis, deren erforderliche Therapie sowie das myelodysplastische Syndrom und die konsekutiv langjährig bestehende Osteoporose sollten aus Sicht der Autoren als komplizierende Faktoren beachtet werden. Angesichts der langjährigen antiresorptiven Vorbehandlung in Verbindung mit der für die Knochenregeneration kritischen Glukokortikoid- [7] und MTX- [12] Therapie, erscheint pathophysiologisch eine osteoanabole Therapiesequenz zunächst naheliegend, wenngleich die Datenlage dahingehend inkonsistent ist [6]. Angesichts des Risikos einer Progression des myelodysplastischen Syndroms im Zuge einer forcierten Zellpropagation unter dem Einsatz von Teriparatid sowie des erhöhten Risikos für kardiovaskuläre Endpunkte bei entsprechendem Ausgangsrisiko unter Romosozumab entschieden wir uns gegen einen solchen Behandlungsansatz.

Durch die Implantation einer inversen Prothese wird die Vorspannung des Musculus deltoideus erhöht. Es scheint denkbar, dass hierdurch auch die Zugbelastung am Ursprung des Muskels an der lateralen Klavikula steigt und damit die Biegelast an der medialen Begrenzung des Muskelursprungs. Diese Stelle befindet sich am lateralen Drittel/Übergang vom mittleren zum lateralen Drittel der Klavikula und somit in der Lokalisation, in der die Klavikulafrakturen beschrieben wurden. Aus Sicht der Autoren sind eine sekundäre Dislokation und Pseudarthrosen unter konservativer Therapie somit wahrscheinlich. Wir entschieden uns daher für die Versorgung mit Plattenosteosynthese. Die laterale Extension der winkelstabilen Platte erlaubte eine multidirektionale Verspannung des lateralen Fragmentes und erhöhte hierdurch nochmals den Ausrisswiderstand. Allerdings erfolgte aufgrund der Frakturmorphologie eine bifragmentäre Schraubenpositionierung in der Frakturzone (• Abb. 5). Möglicherweise hat die hierdurch fehlende Schwingstrecke der Platte die Schraubenbrüche begünstigt. Retrospektiv wäre eine Osteosynthese nach dem Fixateur-interne-Prinzip oder eine zusätzliche Fixation über den Processus coracoideus mit Fadenzugsystem wahrscheinlich überlegen gewesen. Die oben beschriebenen Faktoren, welche als Ursache der Klavikulafraktur nach RSA diskutiert werden, scheinen auch beim Versagen der Osteosynthese eine Rolle zu spielen. Erschwerend kamen im aktuellen Fall die langjährige Osteoporose und die unwillkürlichen Belastungen der Schulter durch einen erhöhten Muskeltonus sowie einen dauerhaften Tremor der oberen Extremitäten bei Morbus Parkinson hinzu.

Die Insuffizienzfraktur der Klavikula stellt eine sehr seltene und komplexe Komplikation nach RSA-Implantation dar. Unabhängig von konservativer oder operativer Versorgung scheinen die Frakturen auch mittelfristig mit Limitationen der Schulterfunktion einherzugehen. In den in der Literatur beschriebenen Fällen zeigten sich keine zufriedenstellenden Ergebnisse unter konservativer Therapie $[1,8,9]$. Dennoch sollte nach Meinung der Autoren die osteosynthetische Versorgung kritisch diskutiert werden. Insbesondere bei limitierter Knochenqualität und Begleiterkrankungen bestehen begrenzte Erfolgsaussichten mit dem Risiko sekundärer Komplikationen [4].

\section{Fazit für die Praxis}

- Die Klavikulafraktur nach Implantation einer inversen Schulterendoprothese ist eine selten beschriebene Komplikation. In den beschriebenen Fällen zeigten sich 
nach konservativer wie auch operativer Therapie negative funktionelle Auswirkungen.

- Die konservative Therapie scheint mit hohen Raten sekundärer Dislokationen einherzugehen.

- Die Indikation zur Osteosynthese sollte aus Sicht der Auroren aber insbesondere bei Begleiterkrankungen wie Osteoporose äußerst kritisch abgewogen werden, da die Gefahr eines Versagens der Osteosynthese mit sekundären Komplikationen besteht.

- Erkrankungen wie Osteoporose sollten vor und nach Prothesenimplantation optimal medikamentös eingestellt werden, um das Frakturrisiko zu senken.

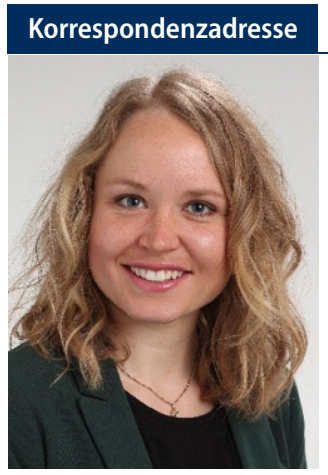

Dr. Laura Elisa Streck

Lehrstuhl für Orthopädie der JuliusMaximilians-Universität Würzburg, Orthpädische Klinik König-Ludwig-Haus Brettreichstr. 11, 97074 Würzburg, Deutschland laura.e.streck@gmail.com

Funding. Open Access funding enabled and organized by Projekt DEAL.

\section{Einhaltung ethischer Richtlinien}

Interessenkonflikt. K. Rueckl übt beratende Tätigkeiten für Stryker und Arthrex aus. Dies hatte keinen Einfluss auf die Inhalte dieses Manuskriptes. L.E. Streck, L. Seefried, F. Genest, T. Reichel und M. Rudert geben an, dass kein Interessenkonflikt besteht.

Die Erstellung des Manuskriptes erfolgte entsprechend der Richtlinien der Deklaration von Helsinki. Für Bildmaterial oder anderweitige Angaben innerhalb des Manuskripts, über die Patienten zu identifizieren sind, liegt von ihnen eine schriftliche Einwilligung vor.

Open Access. Dieser Artikel wird unter der Creative Commons Namensnennung 4.0 International Lizenz veröffentlicht, welche die Nutzung, Vervielfältigung, Bearbeitung, Verbreitung und Wiedergabe in jeglichem Medium und Format erlaubt, sofern Sie den/die ursprünglichen Autor(en) und die Quelle ordnungsgemäß nennen, einen Link zur Creative Commons Lizenz beifügen und angeben, ob Änderungen vorgenommen wurden.

\section{Clavicle stress fracture following reverse shoulder arthroplasty}

We present a rare case of clavicle fracture following reverse shoulder arthroplasty (RSA). This complication may be caused by the higher tension of the deltoid muscle after RSA, similarly to stress fractures of the acromion. Associated bone disease, i.e. osteoporosis, may contribute. In the few case reports available, the affected patients showed significant functional impairments. In the current case, the fracture was addressed with plate fixation. Despite good intraoperative results, osteosynthesis failure with secondary dislocation of the fracture occurred.

\section{Keywords}

Glenohumeral joint · Osteoporosis · Osteosynthesis, fracture · Postoperative complications · Total shoulder replacement
Die in diesem Artikel enthaltenen Bilder und sonstiges Drittmaterial unterliegen ebenfalls der genannten Creative Commons Lizenz, sofern sich aus der Abbildungslegende nichts anderes ergibt. Sofern das betreffende Material nicht unter der genannten Creative Commons Lizenz steht und die betreffende Handlung nicht nach gesetzlichen Vorschriften erlaubt ist, ist für die oben aufgeführten Weiterverwendungen des Materials die Einwilligung des jeweiligen Rechteinhabers einzuholen.

Weitere Details zur Lizenz entnehmen Sie bitte der Lizenzinformation auf http://creativecommons.org/ licenses/by/4.0/deed.de.

\section{Literatur}

1. Anakwenze OA, Pifer MA, Singh A (2014) Clavicle stress fracture after reverse shoulder arthroplasty. J Shoulder Elbow Surg 23(7):e170-2. https://doi. org/10.1016/j.jse.2014.03.015

2. Bohsali Kl, Wirth MA, Rockwood CA (2006) Complications of total shoulder arthroplasty. J Bone Joint Surg Am 88(10):2279-2292. https:// doi.org/10.2106/JBJS.F.00125

3. Collin $P$, Hervé $A$, Walch $G$, Boileau $P$, Muniandy M, Chelli M (2019) Mid-term results of reverse shoulder arthroplasty for glenohumeral osteoarthritis with posterior glenoid deficiency and humeral subluxation. J Shoulder Elbow Surg 28(10):2023-2030. https://doi.org/10.1016/j.jse. 2019.03.002

4. Cornell CN (2003) Internal fracture fixation in patients with osteoporosis. J Am Acad Orthop Surg 11(2):109-119. https://doi.org/10.5435/ 00124635-200303000-00005

5. Favard L, Levigne C, Nerot C, Gerber C, De Wilde L, Mole D (2011) Reverse prostheses in arthropathies with cuff tear. Are survivorship and function maintained over time? Clin Orthop Relat Res 469(9):2469-2475. https://doi.org/10.1007/ s11999-011-1833-y

6. Gorter EA, Reinders CR, Krijnen P, AppelmanDijkstra NM, Schipper IB (2021) The effect of osteoporosis and its treatment on fracture healing a systematic review of animal and clinical studies. Bone Rep 15:101117. https://doi.org/10.1016/j. bonr.2021.101117

7. Hachemi Y, Rapp AE, Picke A-K, Weidinger G, Ignatius A, Tuckermann J (2018) Molecular mechanisms of glucocorticoids on skeleton and bone regeneration after fracture. J Mol Endocrinol
61(1):R75-R90. https://doi.org/10.1530/JME-180024

8. Kim YS, Wang S-I, Lee JH (2016) Clavicle fracture after reverse total shoulder arthroplasty: a case report with literature review. Acta Orthop Traumatol Turc 50(1):111-114. https://doi.org/10. 3944/AOTT.2016.14.0170

9. Mufty S, Milants P, Reynaert P, Mulier T (2016) Clavicular stress fracture following reverse total shoulder arthroplasty: a case report. JBJS Case Connect 6(1):e18. https://doi.org/10.2106/JBJS. CC. 0.00045

10. Otto RJ, Virani NA, Levy JC, Nigro PT, Cuff DJ, Frankle MA (2013) Scapular fractures after reverse shoulder arthroplasty. Evaluation of risk factors and the reliability of a proposed classification. J Shoulder Elbow Surg 22(11):1514-1521. https:// doi.org/10.1016/j.jse.2013.02.007

11. Patterson DC, Chi D, Parsons BO, Cagle PJ (2019) Acromial spine fracture after reverse total shoulder arthroplasty: a systematic review.JShoulderElbow Surg 28(4):792-801. https://doi.org/10.1016/j.jse. 2018.08.033

12. Rolvien T, Jandl NM, Stürznickel J, Beil FT, Kötter I, Oheim R, Lohse AW, Barvencik F, Amling M (2021) Clinical and radiological characterization of patients with immobilizing and progressive stress fractures in methotrexate osteopathy. Calcif Tissue Int 108(2):219-230. https://doi.org/10. 1007/s00223-020-00765-5

13. Singhal K, Rammohan R (2018) Going forward with reverse shoulder arthroplasty. J Clin Orthop Trauma 9(1):87-93. https://doi.org/10.1016/j.jcot. 2017.10.002

14. Wall B, Nove-Josserand L, O'Connor DP, Edwards TB, Walch G(2007) Reverse total shoulderarthroplasty. A review of results according to etiology. J Bone Joint Surg Am 89(7):1476-1485. https://doi.org/ 10.2106/JBJS.F.00666 